\title{
Self-force on moving electric and magnetic dipoles: Dipole radiation, Vavilov-Čerenkov radiation, friction with a conducting surface, and the Einstein-Hopf effect
}

\author{
Kimball A. Milton $\odot,{ }^{1, *}$ Hannah Day, ${ }^{1, \dagger}$ Yang Li, ${ }^{1,2, \star}$ Xin Guo, ${ }^{1, \S}$ and Gerard Kennedy $\odot^{3, \|}$ \\ ${ }^{1}$ H. L. Dodge Department of Physics and Astronomy, University of Oklahoma, Norman, Oklahoma 73019, USA \\ ${ }^{2}$ Department of Physics, Nanchang University, Nanchang 330031, China \\ ${ }^{3}$ School of Mathematical Sciences, University of Southampton, Southampton, SO17 1BJ, United Kingdom
}

(Received 27 June 2020; accepted 5 November 2020; published 9 December 2020)

\begin{abstract}
The classical electromagnetic self-force on an arbitrary time-dependent electric or magnetic dipole moving with constant velocity in vacuum, and in a medium, is considered. Of course, in vacuum there is no net force on such a particle. Rather, because of loss of mass by the particle due to radiation, the self-force precisely cancels this inertial effect, and thus the spectral distribution of the energy radiated by dipole radiation is deduced without any consideration of radiation fields or of radiation reaction, in both the nonrelativistic and relativistic regimes. If the particle is moving in a homogeneous medium faster than the speed of light in the medium, Vavilov-Čerenkov radiation results. This is derived for the different polarization states, in agreement with the earlier results of Frank. The friction experienced by a point (time-independent) dipole moving parallel to an imperfectly conducting surface is examined. Finally, the relativistic quantum/thermal Einstein-Hopf effect is rederived. We obtain a closed form for the spectral distribution of the force and demonstrate that, even if the atom and the blackbody background have independent temperatures, the force is indeed a drag when the imaginary part of the polarizability is proportional to a power of the frequency. The unifying theme of these investigations is that friction on an atom requires a dissipative mechanism, be it through radiation or resistivity in the environment.
\end{abstract}

DOI: 10.1103/PhysRevResearch.2.043347

\section{INTRODUCTION}

Quantum electrodynamic friction has a long history. It is typically referred to as Casimir or quantum friction, and occurs when a polarizable object or atom moves parallel to another such body. For a review with many references, see Ref. [1].

But frictional drag also occurs in the classical regime. Recently, we considered the friction on a charged particle passing parallel to a metallic surface described by the Drude model [2]. We have now investigated the analogous effect when the particle is neutral, but carries an electric or a magnetic dipole moment. We report our results here. We further show how quantum friction emerges, even if the particle does not carry an intrinsic dipole moment, by considering fluctuational averages of dipole moments and electromagnetic fields.

In the course of this investigation, we discovered that, even if the metallic surface is not present, that is, if the particle

\footnotetext{
*kmilton@ou.edu

†hannah.j.day-1@ou.edu

‡leon@ncu.edu.cn

§guoxinmike@ou.edu

"g.kennedy@soton.ac.uk
}

Published by the American Physical Society under the terms of the Creative Commons Attribution 4.0 International license. Further distribution of this work must maintain attribution to the author(s) and the published article's title, journal citation, and DOI. is moving with constant velocity in vacuum, a classical finite self-force can arise. This seems rather different from the Einstein-Hopf effect [3] due to thermal fluctuations in the electromagnetic field, or the Boyer effect [4] due to zero-point fluctuations. The effect we observe does not, of course, mean that the particle slows down (because the configuration can be obtained from that of the particle at rest by a Lorentz transformation). Rather, when the dipole moment possesses time dependence, the radiation produced by the dipole decreases the mass of the particle slightly, so the corresponding decrease in the particle's (relativistic) momentum, transferred to the momentum of the radiation field, is interpreted as the impulse of a drag force. In fact, this force is proportional to the total energy radiated by the dipole. That is, the formula for the energy spectrum emitted by dipole radiation, or equivalently, the radiation reaction force, is derived entirely from the Lorentz force law and the Maxwell-Heaviside equations, without any explicit reference to radiation fields. The physics behind this effect was also noted in a less general context in Ref. [5].

If the vacuum is replaced by a homogeneous medium of permittivity $\varepsilon(\omega)$, and the particle moves at a speed $v$ faster than the speed of light in the medium $1 / \sqrt{\varepsilon}$, VavilovČerenkov radiation is produced. This was worked out for electric and magnetic dipoles many years ago by Frank [6], although there seemed to be some ambiguity in the case of a magnetic dipole [7]. (For a fascinating historical discussion, see Ref. [8].) We discuss these ambiguities here by doing direct calculations using our formalism.

Then, we return to the original problem, that of a neutral particle, possessing either an electric or a magnetic dipole 
moment, moving parallel to an imperfectly conducting surface. Because of dissipation in the material, the particle experiences a frictional force, which depends on the direction of orientation of the dipole. (Our formalism also allows us to derive the friction due to induced Vavilov-Čerenkov radiation in the material when the velocity of the particle exceeds the speed of light in the substrate [9]. This will be described elsewhere.) Although our approach is general, we limit our detailed results to a time-independent dipole, in the low-velocity limit, which is the regime most likely to be accessible to observation.

Finally, we begin our foray into the quantum vacuum regime by quantizing our system, replacing the product of dipole moments by its fluctuational average using the fluctuation-dissipation theorem. Field fluctuations are similarly considered. The force is equivalent to that given by Dedkov and Kyasov [10] and Volokitin and Persson [11], which reduces to the Einstein-Hopf effect [3] in the nonrelativistic limit.

Although the calculations presented here may seem somewhat disparate, and to a large extent reproduce known results, there is a clear commonality in the physics of a moving dipole in a nontrivial background, including the vacuum. Our purpose, in this methodological paper, is to show that all these frictional effects have a common basis, and can be obtained from a simple, relativistic formulation following directly from the Heaviside-Maxwell equations. The interplay between radiation reaction, Vavilov-Čerenkov radiation in the environment, and dissipation in the particle and the environment imply interesting synergic effects. We hope that our investigations spur experimental studies of such phenomena.

The outline of this paper is as follows. In the next section we give general expressions for the self-force on a moving dipole. In Sec. II A we consider the simplest case, where an electric dipole, moving through the vacuum, is oriented in the same direction as the motion, while perpendicular orientation is treated in Sec. II B. The interpretation in terms of dipole radiation is given in Sec. IIC. In Sec. IID it is shown that equivalent formulas are obtained for a moving magnetic dipole. The relation between the currents we use and the electric and magnetic polarizations are also given there. In Sec. III we consider Vavilov-Čerenkov radiation in a uniform dielectric medium due to a moving time-independent electric or magnetic dipole, and obtain, using our machinery, the energy loss rate, or frictional force, and the corresponding energy spectrum, first derived by Frank in 1942 [6], and, in particular, confirm the second form proposed by Frank in 1984 [7] (and see earlier references given there). In Sec. IV we examine the classical friction experienced by a time-independent dipole moving parallel to an imperfectly conducting plate, complementing Ref. [2]. Finally, in Sec. V, we use the fluctuation-dissipation theorem to connect the corresponding quantum friction in vacuum to the Einstein-Hopf effect. Concluding remarks follow in Sec. VI. In Appendix A we show how the current densities for a moving time-dependent dipole are obtained by a Lorentz transformation from those in the rest frame. In Appendix B we give some properties of the Green's functions used, while in Appendix $\mathrm{C}$ we illustrate how our method reproduces the usual static Casimir-Polder force in general. Appendix D describes how the blackbody spectrum appears in a moving frame. Appendix E demonstrates that the relativistic Einstein-Hopf friction is indeed a drag force when the imaginary part of the polarizability is a power law in the frequency.

In this paper we use Heaviside-Lorentz (rationalized) electromagnetic units, and set $c=\hbar=1$.

\section{SELF-FORCE ON DIPOLE}

The force is computed from the Lorentz law for the force density,

$$
\mathbf{f}(\mathbf{r}, t)=\rho(\mathbf{r}, t) \mathbf{E}(\mathbf{r}, t)+\mathbf{j}(\mathbf{r}, t) \times \mathbf{B}(\mathbf{r}, t) .
$$

For a time-dependent electric dipole moving with constant velocity $\mathbf{v}=\hat{\mathbf{x}} v$ in the $x$ direction, the charge and current densities are

$$
\begin{aligned}
\rho(\mathbf{r}, t)= & -\mathbf{d}(t) \cdot \nabla \delta(x-v t) \delta(y) \delta(z), \\
\mathbf{j}(\mathbf{r}, t)= & -\mathbf{v} \mathbf{d}(t) \cdot \nabla \delta(x-v t) \delta(y) \delta(z) \\
& +\dot{\mathbf{d}}(t) \delta(x-v t) \delta(y) \delta(z) .
\end{aligned}
$$

Here $\mathbf{d}(t)$ is the dipole moment in the moving (lab) frame. The relation between the dipole moment in the moving frame and that in the rest frame is given by (2.24) below. This current is that obtained by a Lorentz boost from the charge density of a time-dependent dipole at rest. (See Appendix A.) The second term in the current density arises because the dipole moment is assumed time dependent, so it is required by current conservation:

$$
\frac{\partial}{\partial t} \rho+\nabla \cdot \mathbf{j}=0
$$

(Adding a curl term to $\mathbf{j}$ in the rest frame would correspond to an intrinsic magnetic dipole moment. See Sec. II D.) In the following, it will be convenient to use the frequency Fourier transform of these quantities:

$$
\begin{aligned}
\rho(\mathbf{r} ; \omega)= & -\frac{1}{v} \nabla \cdot\left[\mathbf{d}\left(\frac{x}{v}\right) e^{i \omega x / v} \delta(y) \delta(z)\right], \\
\mathbf{j}(\mathbf{r} ; \omega)= & -\frac{\mathbf{v}}{v} \nabla \cdot\left[\mathbf{d}\left(\frac{x}{v}\right) e^{i \omega x / v} \delta(y) \delta(z)\right] \\
& +\frac{d}{d x} \mathbf{d}\left(\frac{x}{v}\right) e^{i \omega x / v} \delta(y) \delta(z) .
\end{aligned}
$$

In the frequency domain, the electric field can be expressed in terms of the Green's function,

$$
\mathbf{E}(\mathbf{r} ; \omega)=-\frac{1}{i \omega} \int\left(d \mathbf{r}^{\prime}\right) \boldsymbol{\Gamma}\left(\mathbf{r}, \mathbf{r}^{\prime} ; \omega\right) \cdot \mathbf{j}\left(\mathbf{r}^{\prime} ; \omega\right) .
$$

It will be convenient to adopt the transverse Fourier representation

$$
\boldsymbol{\Gamma}\left(\mathbf{r}, \mathbf{r}^{\prime} ; \omega\right)=\int \frac{\left(d \mathbf{k}_{\perp}\right)}{(2 \pi)^{2}} e^{i \mathbf{k}_{\perp} \cdot\left(\mathbf{r}-\mathbf{r}^{\prime}\right)_{\perp}} \mathbf{g}\left(z, z^{\prime} ; \mathbf{k}_{\perp}, \omega\right),
$$

because we have in mind, as treated in Sec. IV, motion next to a surface in the $x-y$ plane. This assumes the system has translational invariance in $x$ and $y$ directions, for which we have the breakup into transverse electric (TE or $E$ ) and transverse magnetic (TM or $H$ ) modes, with $k^{2}=\mathbf{k}_{\perp}^{2}$, in a dielectric 
medium with permittivity $\varepsilon(z, \omega)$ :

$$
\mathbf{g}=\left(\begin{array}{ccc}
\frac{k_{x}^{2}}{k^{2}} \frac{1}{\varepsilon \varepsilon^{\prime}} \partial_{z} \partial_{z^{\prime}} g^{H}+\frac{k_{y}^{2}}{k^{2}} \omega^{2} g^{E} & \frac{k_{x} k_{y}}{k^{2}}\left(\frac{1}{\varepsilon \varepsilon^{\prime}} \partial_{z} \partial_{z^{\prime}} g^{H}-\omega^{2} g^{E}\right) & i k_{x} \frac{1}{\varepsilon \varepsilon^{\prime}} \partial_{z} g^{H} \\
\frac{k_{x} k_{y}}{k^{2}}\left(\frac{1}{\varepsilon \varepsilon^{\prime}} \partial_{z} \partial_{z^{\prime}} g^{H}-\omega^{2} g^{E}\right) & \frac{k_{y}^{2}}{k^{2}} \frac{1}{\varepsilon \varepsilon^{\prime}} \partial_{z} \partial_{z^{\prime}} g^{H}+\frac{k_{x}^{2}}{k^{2}} \omega^{2} g^{E} & i k_{y} \frac{1}{\varepsilon \varepsilon^{\prime}} \partial_{z} g^{H} \\
-i k_{x} \frac{1}{\varepsilon \varepsilon^{\prime}} \partial_{z^{\prime}} g^{H} & -i k_{y} \frac{1}{\varepsilon \varepsilon^{\prime}} \partial_{z^{\prime}} g^{H} & k^{2} \frac{1}{\varepsilon \varepsilon^{\prime}} g^{H}
\end{array}\right),
$$

where $\varepsilon=\varepsilon(z, \omega)$ and $\varepsilon^{\prime}=\varepsilon\left(z^{\prime}, \omega\right)$. Here we specialize to the vacuum case, $\varepsilon=1$, but we will generalize in Sec. III. Here the transverse electric and transverse magnetic Green's functions are equal in vacuum,

$$
g^{E}\left(z, z^{\prime} ; \mathbf{k}_{\perp}, \omega\right)=g^{H}\left(z, z^{\prime} ; \mathbf{k}_{\perp}, \omega\right)=\frac{1}{2 \kappa} e^{-\kappa\left|z-z^{\prime}\right|},
$$

with $\kappa=\sqrt{k_{x}^{2}+k_{y}^{2}-\omega^{2}}$, which is, in general, complex.

To compute the Lorentz force on the moving dipole, we first eliminate, by use of Eq. (2.3), the charge density from the time-averaged force density in Eq. (2.1), integrated over all space,

$$
\begin{aligned}
\overline{\mathbf{F} T} & =\int \frac{d \omega}{2 \pi} \int(d \mathbf{r})\left[\rho(\mathbf{r} ; \omega)^{*} \mathbf{E}(\mathbf{r} ; \omega)+\mathbf{j}(\mathbf{r} ; \omega)^{*} \times \mathbf{B}(\mathbf{r} ; \omega)\right] \\
& =\int \frac{d \omega}{2 \pi} \int(d \mathbf{r})\left(d \mathbf{r}^{\prime}\right) \frac{1}{\omega^{2}} j_{i}(\mathbf{r} ; \omega)^{*} \nabla \Gamma_{i k}\left(\mathbf{r}, \mathbf{r}^{\prime} ; \omega\right) j_{k}\left(\mathbf{r}^{\prime} ; \omega\right) \equiv \operatorname{Tr} \frac{1}{\omega^{2}} \mathbf{j}^{*}(\nabla) \boldsymbol{\Gamma} \mathbf{j}
\end{aligned}
$$

The last form is a matrix notation where the trace Tr includes the integration over coordinates and frequency. Here, $T$ is the (large) time that the configuration exists. This form of the mean force, including both the electric and magnetic terms, is reminiscent of the expression used in quantum mechanics [12]. Now inserting the current densities (2.4b) into this, we obtain for the force in the direction of motion, taking advantage of the $\delta$ functions,

$$
\begin{aligned}
\overline{\mathbf{F}_{x}} T= & \left.\int \frac{d \omega}{2 \pi} \int d x d x^{\prime} e^{-i \omega x / v} \frac{1}{v}\left[\mathbf{d}\left(\frac{x}{v}\right) \cdot \nabla \mathbf{v}+\dot{\mathbf{d}}\left(\frac{x}{v}\right)\right] \cdot \frac{1}{\omega^{2}} \int \frac{\left(d \mathbf{k}_{\perp}\right)}{(2 \pi)^{2}} e^{i k_{x}\left(x-x^{\prime}\right)} i k_{x} \mathbf{g}\left(z, z^{\prime} ; \mathbf{k}_{\perp}, \omega\right)\right|_{z=z^{\prime}} \\
& \cdot \frac{1}{v}\left[\mathbf{v} \overleftarrow{\nabla}^{\prime} \cdot \mathbf{d}\left(\frac{x^{\prime}}{v}\right)+\dot{\mathbf{d}}\left(\frac{x^{\prime}}{v}\right)\right] e^{i \omega x^{\prime} / v} .
\end{aligned}
$$

Here, we have integrated by parts, and the minus sign obtained from so doing is incorporated in the differential operator $\overleftarrow{\nabla}^{\prime}$ In the transverse directions, the gradient operators are to be interpreted as $\nabla_{\perp}=i \mathbf{k}_{\perp}$ and $\nabla_{\perp}^{\prime}=-i \mathbf{k}_{\perp}$. Similarly, the $\dot{\mathbf{d}}$ term can be integrated by parts, yielding a factor $i\left(\omega-k_{x} v\right)$. Now, carrying out the integrations over $x$ and $x^{\prime}$ leads to the Fourier transform of the dipole moment,

$$
\tilde{\mathbf{d}}(\omega)=\int_{-\infty}^{\infty} d t e^{i \omega t} \mathbf{d}(t)
$$

so the product of dipole moments appears as $\tilde{\mathbf{d}}(\omega-$ $\left.v k_{x}\right)^{*} \tilde{\mathbf{d}}\left(\omega-v k_{x}\right)$.

\section{A. d || $\mathbf{v}$}

Suppose first the dipole is polarized parallel to the motion, that is, $\mathbf{d}$ and $\mathbf{v}$ are both in the $x$ direction. Then, from Eqs. (2.10) and (2.7), the force in the $x$ direction is

$$
\overline{F_{x}^{\|}} T=\frac{i}{8 \pi^{3}} \int_{-\infty}^{\infty} d \omega \int d k_{x} d k_{y}\left|\tilde{d}\left(\omega-v k_{x}\right)\right|^{2} k_{x} \frac{\left(\omega^{2}-k_{x}^{2}\right)}{2 \kappa} .
$$

The real part of the integral is zero, because the integrand is odd under the substitutions $\omega \rightarrow-\omega$ and $k_{x} \rightarrow-k_{x}$. The imaginary part of the integrand can only arise from $\kappa$, which becomes imaginary when $k^{2}<\omega^{2}$. The appropriate branch of the square root is determined by the requirement that the singularities lie in the lower-half $\omega$ plane, since we are dealing with the retarded propagator. Then

$$
\omega^{2}>k^{2}: \quad \sqrt{k^{2}-\omega^{2}}=-i \operatorname{sgn}(\omega) \sqrt{\omega^{2}-k^{2}} .
$$

When $\omega^{2}-k_{x}^{2}>0$, the $k_{y}$ integral is simply

$$
\int_{-\sqrt{\omega^{2}-k_{x}^{2}}}^{\sqrt{\omega^{2}-k_{x}^{2}}} \frac{d k_{y}}{\sqrt{\omega^{2}-k_{x}^{2}-k_{y}^{2}}}=\pi
$$

and we are left with

$$
\overline{F_{x}^{\|}} T=-\frac{1}{16 \pi^{2}} \int d \omega d k_{x}\left|\tilde{d}\left(\omega-v k_{x}\right)\right|^{2} \operatorname{sgn}(\omega) k_{x}\left(\omega^{2}-k_{x}^{2}\right) .
$$

When we change variables by writing $v=\omega-k_{x} v$ and $k_{x}=$ $u v$, we obtain

$$
\begin{aligned}
\overline{F_{x}^{\|}} T= & -\frac{1}{16 \pi^{2}} \int_{-\infty}^{\infty} d v v^{4}|\tilde{d}(v)|^{2} \int_{-1 /(1+v)}^{1 /(1-v)} d u \\
& \times u\left[(1+v u)^{2}-u^{2}\right] \\
= & -\frac{v}{12 \pi^{2}} \gamma^{6} \int_{-\infty}^{\infty} d v v^{4}|\tilde{d}(v)|^{2} .
\end{aligned}
$$

Here $\gamma=\left(1-v^{2}\right)^{-1 / 2}$ is the usual relativistic dilation factor.

\section{B. $d \perp v$}

If the polarization of the dipole is perpendicular to the motion, say in the $y$ direction, we again start from Eq. (2.10). 
Following the same procedure we detailed in the previous subsection, and inserting the appropriate components of the reduced Green's function from Eq. (2.7), we find, after some algebra, for the drag force in the direction of motion

$$
\begin{aligned}
\overline{F_{x}{ }^{\perp}} T= & v^{2} \int \frac{d \omega\left(d \mathbf{k}_{\perp}\right)}{(2 \pi)^{3}}\left|\tilde{d}\left(\omega-v k_{x}\right)\right|^{2}\left(-\frac{k_{x}}{2 i \kappa}\right) \\
& \times\left[k_{y}^{2}\left(1-\frac{1}{v^{2}}\right)+\left(k_{x}-\frac{\omega}{v}\right)^{2}\right] .
\end{aligned}
$$

Again, carrying out the integral on $k_{y}$ above the branch line of the square root, and making the same changes of variables as before, we find

$$
\begin{aligned}
\overline{F_{x}^{\perp}} T= & -\frac{v^{2}}{16 \pi^{2}} \int_{-\infty}^{\infty} d v|\tilde{d}(v)|^{2} v^{4} \\
& \times \int_{-1 /(1+v)}^{1 /(1-v)} d u u \frac{1}{2}\left\{\frac{1}{v^{2}}+\left[1+v u\left(1-\frac{1}{v^{2}}\right)\right]^{2}\right\} \\
= & -\frac{v}{12 \pi^{2}} \gamma^{4} \int_{-\infty}^{\infty} d v v^{4}|\tilde{d}(v)|^{2} .
\end{aligned}
$$

\section{Interpretation and discussion}

In the above two subsections, we have performed straightforward calculations of the classical electromagnetic selfforce on an arbitrary time-varying electric dipole undergoing uniform motion. The results are slightly different depending on the orientation of the dipole. If the dipole is perpendicular (parallel) to the motion, the time-averaged force in the direction of the motion is

$$
\left.\frac{\overline{F_{x}^{\perp}}}{F_{x}^{\|}}\right\}=-\frac{v}{12 \pi^{2} T}\left\{\begin{array}{l}
\gamma^{4} \\
\gamma^{6}
\end{array}\right\} \int_{-\infty}^{\infty} d v v^{4}|\tilde{d}(v)|^{2},
$$

in terms of the Fourier transform of the dipole moment. In the above we assumed that the direction of the dipole moment was fixed either parallel or perpendicular to the direction of motion. For general orientation, the off-diagonal elements of the reduced Green's dyadic are either odd in $k_{y}$ or proportional to $\operatorname{sgn}\left(z-z^{\prime}\right)$, which vanishes in the coincident limit. Thus we have in general

$$
\overline{F_{x}}=-\frac{v}{12 \pi^{2} T} \gamma^{4} \int_{-\infty}^{\infty} d v v^{4}\left[\left|\tilde{\mathbf{d}}_{\perp}(v)\right|^{2}+\gamma^{2}\left|\tilde{\mathbf{d}}_{\|}(v)\right|^{2}\right],
$$

in terms of the dipole moment components perpendicular (parallel) to the direction of motion.

Three observations immediately jump out.

(i) For an undamped oscillator of frequency $\omega_{0}$, the integral over $v$ is proportional to

$$
2 \pi \delta(0)=\left.\lim _{T \rightarrow \infty} \int_{-T / 2}^{T / 2} d t e^{i \omega t}\right|_{\omega=0}=T,
$$

because $\tilde{d}(v) \propto \delta\left(v-\omega_{0}\right)$.

(ii) In the nonrelativistic limit the drag forces are identical.

(iii) The friction is proportional to the total energy radiated by an oscillating dipole at rest [13]

$$
E_{R}^{\prime}=\frac{1}{12 \pi^{2}} \int_{-\infty}^{\infty} d v v^{4}\left|\tilde{\mathbf{d}}^{\prime}(v)\right|^{2},
$$

where the integrand is the spectral energy distribution of a radiating dipole. (Primes denote quantities in the particle's rest frame.)

Thus, for low velocities, the force satisfies

$$
\overline{F_{x}} T=-v E_{R}^{\prime} .
$$

This result has an extremely simple interpretation. Of course, there is no force on a uniformly moving dipole in vacuum, since it may be obtained from a dipole at rest by a Lorentz boost. But, because the dipole loses energy $E_{R}^{\prime}$ over the course of its motion, its mass decreases accordingly, and therefore, nonrelativistically, its momentum decreases by $E_{R}^{\prime} v$. This is the negative of the momentum carried off by the radiation field. Momentum conservation means that a radiation reaction force $\overline{F_{x}}=-E_{R}^{\prime} v / T$ is acting on the moving dipole.

In the relativistic regime, the different factors of $\gamma$ are easily understood. One factor of $\gamma$ comes from the Lorentz transformation of the momentum of the radiated energy, $\left(P_{R}\right)_{x}=v \gamma E_{R}^{\prime}$. Then if we transform the dipole moments in the moving frame to those in the rest frame,

$$
\gamma d_{x}(\gamma t)=d_{x}^{\prime}(t), \quad d_{y}(\gamma t)=d_{y}^{\prime}(t),
$$

the Fourier transforms are related by

$$
\tilde{d}_{x}\left(\frac{v}{\gamma}\right)=\tilde{d}_{x}^{\prime}(v), \quad \frac{1}{\gamma} \tilde{d}_{y}\left(\frac{v}{\gamma}\right)=\tilde{d}_{y}^{\prime}(v) .
$$

Thus when $P_{R}$ is expressed in terms of the dipole moment in the moving frame $\mathbf{d}$, we obtain exactly the structure seen in Eq. (2.19). In terms of the dipole moment in the rest frame of the particle, the average force is given by

$$
\overline{F_{x}}=-\frac{v \gamma}{6 \pi^{2} T} \int_{0}^{\infty} d v v^{4}\left|\tilde{\mathbf{d}}^{\prime}(v)\right|^{2} .
$$

This effect, nonrelativistically, was seen in Ref. [5] in the special case of a two-level system with transition frequency $\omega_{0}$. This is described by a dipole with a single frequency, as mentioned above, $d(\omega)=d_{0} 2 \pi \delta\left(\omega-\omega_{0}\right)$, so from Eq. (2.26)

$$
\mathbf{F}=-\mathbf{v} \frac{\omega_{0}^{4} d_{0}^{2}}{3 \pi},
$$

which is the result (16) of Ref. [5]. The inertial origin of the effect is given in Eq. (17) of that reference. What might not be immediately obvious in that work is that the effect is entirely classical. It further appears that our method is somewhat more straightforward.

\section{Magnetic dipole}

What about a magnetic dipole moving through vacuum? Precisely the same considerations apply, and because the results are obtained by a duality transformation, $\mathbf{d} \rightarrow \boldsymbol{\mu}$ and $g^{H}=g^{E}$ in vacuum, ${ }^{1}$ the same form of the inertial effect

\footnotetext{
${ }^{1}$ In dual electrodynamics, the magnetic current would have the same form as Eq. (2.2b), except $\mathbf{d} \rightarrow \boldsymbol{\mu}$.
} 
emerges:

$$
\begin{aligned}
\overline{F_{x}} & =-\frac{v}{12 \pi^{2} T} \gamma^{4} \int_{-\infty}^{\infty} d v v^{4}\left[\left|\tilde{\boldsymbol{\mu}}_{\perp}(v)\right|^{2}+\gamma^{2}\left|\tilde{\boldsymbol{\mu}}_{\|}(v)\right|^{2}\right] \\
& =-\frac{v \gamma}{12 \pi^{2} T} \int_{-\infty}^{\infty} d v v^{4}\left|\tilde{\boldsymbol{\mu}}^{\prime}(v)\right|^{2},
\end{aligned}
$$

again expressed in terms of the energy loss due to magnetic dipole radiation, where $\tilde{\boldsymbol{\mu}}^{\prime}$ is the dipole moment in the rest frame of the particle.

For the following applications, however, duality will fail, because a dielectric medium breaks the symmetry between electric and magnetic quantities. So we must use the electric current density of a moving time-dependent magnetic dipole, obtained from the current of a magnetic dipole at rest

$$
\mathbf{j}^{\prime}\left(\mathbf{r}^{\prime}, t^{\prime}\right)=\nabla^{\prime} \times \boldsymbol{\mu}^{\prime}\left(t^{\prime}\right) \delta\left(\mathbf{r}^{\prime}\right),
$$

which after a boost of velocity $\mathbf{v}$ yields

$$
\begin{aligned}
& \rho(\mathbf{r}, t)=-[\mathbf{v} \times \boldsymbol{\mu}(t)] \cdot \nabla \delta(\mathbf{r}-\mathbf{v} t), \\
& \mathbf{j}(\mathbf{r}, t)=-\boldsymbol{\mu}(t) \times \nabla \delta(\mathbf{r}-\mathbf{v} t)+\partial_{t}[\mathbf{v} \times \boldsymbol{\mu}(t) \delta(\mathbf{r}-\mathbf{v} t)],
\end{aligned}
$$

where $\boldsymbol{\mu}(t)$ is the magnetic dipole moment in the moving frame. The time derivative term is required by current conservation, Eq. (2.3). It is then straightforward algebra to show this current, inserted into our general construction (2.9), yields the radiation formula (2.28), as asserted.

It actually might seem more natural to pose this problem in terms of electric and magnetic polarizations $\mathbf{P}$ and $\mathbf{M}$, instead of electric currents and charges. Of course, the two are related by

$$
\rho=-\nabla \cdot \mathbf{P}, \quad \mathbf{j}=\nabla \times \mathbf{M}+\frac{\partial}{\partial t} \mathbf{P} .
$$

By inspection of Eqs. (2.2) and (2.30), it is immediately seen that

$$
\begin{aligned}
\mathbf{P}(\mathbf{r}, t) & =[\mathbf{d}(t)-\boldsymbol{\mu}(t) \times \mathbf{v}] \delta(\mathbf{r}-\mathbf{v} t), \\
\mathbf{M}(\mathbf{r}, t) & =[\boldsymbol{\mu}(t)+\mathbf{d}(t) \times \mathbf{v}] \delta(\mathbf{r}-\mathbf{v} t) .
\end{aligned}
$$

\section{VAVILOV-ČERENKOV RADIATION}

The above calculations were performed assuming the background was vacuum. It is easy to extend them to the motion of a dipole through a homogeneous dielectric medium. In this case, a new phenomenon can emerge, when the velocity of the particle exceeds the speed of light in the medium $1 / n$, where the index of refraction is $n(\omega)=\sqrt{\varepsilon(\omega)}$. This is the famous Vavilov-Čerenkov effect, usually considered for a charged particle $[14,15]$.

We repeat the above calculations using the general form of the Green's dyadic (2.7), where now

$$
\begin{aligned}
g^{E}\left(z, z^{\prime}\right) & =\frac{1}{2 \kappa} e^{-\kappa\left|z-z^{\prime}\right|}, \quad g^{H}\left(z, z^{\prime}\right)=\frac{\varepsilon}{2 \kappa} e^{-\kappa\left|z-z^{\prime}\right|}, \\
\kappa & =\sqrt{k^{2}-\omega^{2} \varepsilon(\omega)} .
\end{aligned}
$$

Then,

$$
g_{x x}(0,0)=\frac{1}{2 \kappa}\left[\omega^{2}-\frac{k_{x}^{2}}{\varepsilon(\omega)}\right]
$$

and for a longitudinally polarized electric dipole we have the drag force given by

$$
\begin{aligned}
\overline{F_{x}^{\|}} T= & \frac{1}{16 \pi^{2}} \int_{-\infty}^{\infty} d v v^{3}|v||\tilde{d}(v)|^{2} \int d u u \operatorname{sgn}[v(1+v u)] \\
& \times\left[(1+v u)^{2}-\frac{u^{2}}{\varepsilon[v(1+v u)]}\right]
\end{aligned}
$$

Here, the limits of the $u$ integration are determined by

$$
\omega^{2} \varepsilon(\omega)>k_{x}^{2} \quad \text { or } \quad(1+v u)^{2} \varepsilon[v(1+v u)]>u^{2} .
$$

In the case that $\varepsilon$ is independent of frequency, the limits become

$$
-\frac{1}{1 / n+v}, \quad \frac{1}{1 / n-v},
$$

where $n=\sqrt{\varepsilon}$, and then if the speed of the dipole is smaller than the speed of light in the medium, $v<1 / n$,

$$
\overline{F_{x}^{\|}} T=-v \frac{n^{3}}{\left(1-v^{2} n^{2}\right)^{3}} E_{R}, \quad E_{R}=\frac{1}{12 \pi^{2}} \int_{-\infty}^{\infty} d v v^{4}|\tilde{\mathbf{d}}(v)|^{2},
$$

in terms of the radiated dipole energy $E_{R}$. Similarly, for the transverse polarization, we find

$$
\overline{F_{x}^{\perp}} T=-v \frac{n^{3}}{\left(1-v^{2} n^{2}\right)^{2}} E_{R} .
$$

These are simple generalizations of Eq. (2.19).

The assumption of dispersionless permittivity is quite unrealistic, of course. Instead, assume dispersion, but let us suppose in the relevant frequency region that the index of refraction is real and greater than one. In order to obtain a simple result, let us further consider only the case where the dipole has no time dependence, $\mathbf{d}(t)=\mathbf{d}_{0}$, so there is no radiation in the rest frame. But now there is Vavilov-Cerenkov radiation in the frame where the dipole moves with constant velocity greater than that of the speed of light, $v>1 / n(\omega)$. The $v$ integral in Eq. (3.3) becomes trivial because

$$
\tilde{\mathbf{d}}(\omega)=2 \pi \delta(\omega) \mathbf{d}_{0},
$$

where, again as in Eq. (2.21), we interpret $2 \pi \delta(0)=T$. The result for the drag in the case of a moving electric dipole polarized in the direction parallel to the motion is, after changing variable, $k_{x} v=\omega$,

$$
\left(F_{x}^{\|}\right)_{d}=-\frac{d_{0}^{2}}{4 \pi} \frac{1}{v^{2}} \int_{\omega>0} d \omega \omega^{3}\left[1-\frac{1}{v^{2} n(\omega)^{2}}\right],
$$

where the integration extends over those positive frequencies for which the spectral distribution is positive. This distribution coincides with the result of Frank [6], Eq. (2.33) there. (See also Refs. [7,16].) Note that the force on the particle is the energy loss rate per unit length traveled by the particle, $-\partial E / \partial x$. The frequency integrand is the frequency spectrum of radiated energy. See, for example, Ref. [13], Chap. 36.

The result for perpendicular polarization is somewhat different, but obtained in precisely the same way:

$$
\left(F_{x}^{\perp}\right)_{d}=-\frac{d_{0}^{2}}{8 \pi} \int_{\omega>0} d \omega \omega^{3} n(\omega)^{2}\left[1-\frac{1}{v^{2} n(\omega)^{2}}\right]^{2},
$$


coinciding with the spectral distribution found by Frank [6], Eq. (2.34).

As noted above, in vacuum there is no difference between the formulas for the drag forces for a magnetic dipole as compared to an electric dipole. But this is not the case in the medium. Nevertheless, the steps are just the same, with the duality transformations, $d_{0} \rightarrow \mu_{0}, g^{E} \leftrightarrow g^{H}$, and in the construction (2.7), $\varepsilon \rightarrow \mu=1$ (the latter being the permeability). So for a constant magnetic dipole polarized longitudinally,

$$
\left(F_{x}^{\|}\right)_{\mu}=-\frac{\mu_{0}^{2}}{4 \pi v^{2}} \int_{\omega>0} d \omega \omega^{3} n(\omega)^{2}\left[1-\frac{1}{v^{2} n(\omega)^{2}}\right]
$$

which agrees with Ref. [7], Eq. (4.35), but there seems to be a missing $n^{2}$ in Ref. [16]. For perpendicular polarization, the result, after a bit of algebra, is

$$
\left(F_{x}^{\perp}\right)_{\mu}=-\frac{\mu_{0}^{2}}{8 \pi} \int_{\omega>0} d \omega \omega^{3} n(\omega)^{4}\left[1-\frac{1}{v^{2} n(\omega)^{2}}\right]^{2},
$$

which agrees with one of the two alternatives, Eq. (4.37), given by Ref. [7]. ${ }^{2}$ In fact, the extra $n(\omega)^{2}$ in Eqs. (3.12) and (3.11) compared to Eqs. (3.10) and (3.9) is a trivial conse- quence of Babinet's principle [17], p. 91, the invariance of the Heaviside-Maxwell equations in the frequency domain under

$$
\begin{aligned}
& \mathbf{E}(\mathbf{r} ; \omega) \rightarrow \sqrt{\frac{\mu(\omega)}{\varepsilon(\omega)}} \mathbf{H}(\mathbf{r} ; \omega), \\
& \mathbf{H}(\mathbf{r} ; \omega) \rightarrow-\sqrt{\frac{\varepsilon(\omega)}{\mu(\omega)}} \mathbf{E}(\mathbf{r} ; \omega), \\
& j_{e}^{\nu}(\mathbf{r} ; \omega) \rightarrow \sqrt{\frac{\varepsilon(\omega)}{\mu(\omega)}} j_{m}^{\nu}(\mathbf{r} ; \omega), \\
& j_{m}^{\nu}(\mathbf{r} ; \omega) \rightarrow-\sqrt{\frac{\mu(\omega)}{\varepsilon(\omega)}} j_{e}^{\nu}(\mathbf{r} ; \omega) .
\end{aligned}
$$

However, this treatment is suspect, since the dielectric medium breaks dual symmetry, because it has electric susceptibility, but no magnetic susceptibility. We should repeat the calculation using the electric current (2.30b). Perhaps not surprisingly, the same result (3.11) is obtained for parallel polarization. But that is not the case for perpendicular polarization. Since this is a bit more complicated, and perhaps controversial, let us supply a few more details. Assuming $\boldsymbol{\mu}$ points in the $y$ direction, with the velocity in the $x$ direction, the general formula reduces to

$$
\begin{aligned}
\left(\overline{F_{x}^{\perp}}\right)_{\mu} T & =\int \frac{d \omega d k_{x} d k_{y}}{(2 \pi)^{3}} \frac{i k_{x}}{\omega^{2}}\left|\tilde{\mu}\left(\omega-v k_{x}\right)\right|^{2}\left[\partial_{z} \partial_{z^{\prime}} g_{x x}+i\left(k_{x}-v \omega\right)\left(\partial_{z} g_{x z}-\partial_{z^{\prime}} g_{z x}\right)+\left(k_{x}-v \omega\right)^{2} g_{z z}\right] \\
& =T \frac{\mu_{0}^{2}}{8 \pi^{2}} \int d k_{x} d k_{y} \frac{i k_{x}}{\kappa}\left[\left(v k_{x}\right)^{2}\left(\sqrt{\varepsilon}-\frac{1}{\sqrt{\varepsilon}}\right)^{2}+k_{y}^{2}\left(\frac{v^{2}}{\varepsilon}-1\right)\right] .
\end{aligned}
$$

Here, we have assumed the dipole has no time variation, $\boldsymbol{\mu}(t)=\hat{\mathbf{y}} \mu_{0}$. Carrying out the $k_{y}$ integration as before, we obtain the result (letting $v k_{x}=\omega$ )

$$
\left(F_{x}^{\perp}\right)_{\mu}=-\frac{\mu_{0}^{2}}{8 \pi v^{2}} \int_{\omega>0} d \omega \omega^{3} n^{2}(\omega)\left[2\left(1-\frac{1}{n(\omega)^{2}}\right)^{2}-\left(1-\frac{v^{2}}{n(\omega)^{2}}\right)\left(1-\frac{1}{v^{2} n(\omega)^{2}}\right)\right] .
$$

The integral is over those positive frequencies for which $n(\omega) v>1$. The integrand is the spectral distribution of Vavilov-Čerenkov radiation found by Frank in Ref. [7], Eq. (4.36). It is somewhat surprising because it is discontinuous at threshold; that is, it is zero if $v n<1$, but it jumps to a finite value when the particle exceeds the speed of light for a given frequency. The earlier result (3.12), obtained by the plausible duality argument, is indeed incorrect.

In fact, the issue is quite subtle: the duality argument and, therefore, Eq. (3.12) is correct for a "Gilbert" magnetic dipole composed of a magnetic monopole-antimonopole pair, but for an "Ampèrian" dipole due to electric currents Eq. (3.15) is correct. This is discussed in detail in Ref. [8]; see also Refs. $[18,19]$. On the other hand, for the special case of a dipole oriented in the direction parallel to the motion, it is

\footnotetext{
${ }^{2}$ It might be noted that Eq. (3.9) is obtained from Eq. (3.10) by differentiating with respect to $\ln v^{2}$. The same is true for Eqs. (3.11) and (3.12).
}

straightforward to show that the duality argument is valid, and hence Eq. (3.11) holds in either description.

The dipolar Vavilov-Čenenkov effect is rather small. Compared to the corresponding charged particle effect, as described, for example, in Refs. [2,13], where the corresponding frictional force is

$$
\left(F_{x}\right)_{e}=-\frac{e^{2}}{4 \pi} \int d \omega \omega\left(1-\frac{1}{v^{2} n(\omega)^{2}}\right),
$$

the electric dipole effect is smaller by a factor of order $[d /(e \lambda)]^{2}$, where $\lambda$ is the characteristic wavelength of the radiation emitted. This is typically a very small number, because the size of the particle is small compared to an optical wavelength.

\section{FRICTION OF DIPOLE PASSING CLOSE TO CONDUCTING SURFACE}

Next, we consider either an electric or a magnetic dipole moving in vacuum with constant velocity parallel to an imperfectly conducting surface in the $x-y$ plane. The idea is an 
immediate generalization of the analysis of the same situation for a charged particle [2]. We start with Eq. (2.10), which for a time-independent electric dipole $\mathbf{d}(t)=\mathbf{d}_{0}$ yields

$$
\left(F_{x}\right)_{d}=\frac{d_{0}^{2} v^{2}}{4 \pi^{2}} \int d k_{x} d k_{y} \frac{i k_{x}}{\omega^{2}} \nabla_{n} \nabla_{n}^{\prime} g_{x x}, \quad \omega=v k_{x},
$$

where $\nabla_{n}=\hat{\mathbf{n}} \cdot \nabla$, with $\hat{\mathbf{n}}=\mathbf{d}_{0} / d_{0}$. Here $g_{x x}$ is given in Eq. (2.7) with $\varepsilon=1$, but now

$$
g^{E, H}=\frac{1}{2 \kappa}\left[e^{-\kappa\left|z-z^{\prime}\right|}+r^{E, H} e^{-\kappa\left(z+z^{\prime}\right)}\right], \quad z, z^{\prime}>0,
$$

in the vacuum region above the conductor, which occupies the semispace $z<0$, where the reflection coefficients are

$$
\begin{aligned}
r^{E} & =\frac{\kappa-\kappa^{\prime}}{\kappa+\kappa^{\prime}}, \quad r^{H}=\frac{\kappa-\kappa^{\prime} / \varepsilon}{\kappa+\kappa^{\prime} / \varepsilon}, \\
\kappa & =\sqrt{k^{2}-\omega^{2}}, \quad \kappa^{\prime}=\sqrt{k^{2}-\omega^{2} \varepsilon} .
\end{aligned}
$$

The required imaginary part can only come from the reflection coefficients, because $\kappa$ is real. It is convenient to write the above integral in polar coordinates, with $k_{x}=\gamma \kappa \cos \theta$ and $k_{y}=\kappa \sin \theta$.

Depending on the polarization of the dipole relative to the surface and the direction of motion, the force can be written in terms of transverse electric and transverse magnetic contributions:

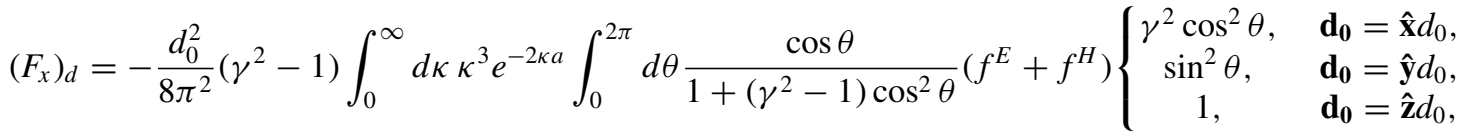

where $a$ is the distance between the trajectory of the dipole and the surface. Here $f^{E, H}$ are the same functions appearing in Ref. [2]:

$$
\begin{aligned}
& f^{E}(\kappa, \theta, \gamma)=2 \sin ^{2} \theta \operatorname{Im}\left[1+\sqrt{1-\left(\gamma^{2}-1\right)(\varepsilon-1) \cos ^{2} \theta}\right]^{-1} \\
& f^{H}(\kappa, \theta, \gamma)=2 \frac{\gamma^{2}}{\gamma^{2}-1} \operatorname{Im}\left[1+\frac{1}{\varepsilon} \sqrt{1-\left(\gamma^{2}-1\right)(\varepsilon-1) \cos ^{2} \theta}\right]^{-1}
\end{aligned}
$$

We model the conductor by the Drude model $^{3}$ :

$$
\varepsilon(\omega)=1-\frac{\omega_{p}^{2}}{\omega^{2}+i \nu \omega},
$$

in terms of the plasma frequency $\omega_{p}$ and the damping parameter $v$.

Because the $f$ 's are the same as discussed previously, we can carry over various limits from Ref. [2]. We will content ourselves here with the low-velocity limit, $v \ll v a \ll 1$. Defining $\hat{\alpha}=2 \omega_{p} a, \hat{\beta}=2 v a$, and $\hat{u}=2 \kappa a$, we have [2]

$$
f^{E}=\frac{\hat{\alpha}^{2} v}{4 \hat{u} \hat{\beta}} \cos \theta \sin ^{2} \theta, \quad f^{H}=\frac{2 \hat{u} \hat{\beta}}{\hat{\alpha}^{2} v} \cos \theta, \quad v \ll \hat{\beta} \ll 1 .
$$

Obviously, the TE contribution is subdominant, and the leading behavior is

$$
\left(F_{x}^{x, y, z}\right)_{d} \approx-\frac{3 d_{0}^{2}}{32 \pi a^{4}} \frac{\hat{\beta} v}{\hat{\alpha}^{2}}(3,1,4), \quad v \ll \hat{\beta} \ll 1,
$$

where the superscript refers to the direction of polarization of $\mathbf{d}_{0}$. If this is averaged over the three polarizations, we obtain the force on a "tumbling" dipole, expressed in terms of the static conductivity, $\sigma=\omega_{p}^{2} /(4 \pi v)$,

$$
F_{x}=-\frac{1}{32 \pi^{2}} \frac{d_{0}^{2}}{\sigma a^{5}} .
$$

\footnotetext{
${ }^{3}$ In this case the imaginary part only comes from that of $\varepsilon(\omega)$. Even if $\varepsilon$ is real, but $v>1 / \sqrt{\varepsilon(\omega)}$, we will encounter an imaginary part from the induced Vavilov-Čerenkov effect [9]. We will examine this situation in detail elsewhere.
}

This is the result given in Ref. [20].

The magnetic friction is derived similarly. We use the current $(2.30 \mathrm{~b})$ to write the force as $\left(\omega=v k_{x}\right)$

$\left(F_{x}\right)_{\mu}=\int \frac{d k_{x} d k_{y}}{(2 \pi)^{2}} \frac{i k_{x}}{\omega^{2}} \boldsymbol{\mu}_{0} \times(\nabla-i \mathbf{v} \omega) \cdot \mathbf{g} \cdot \boldsymbol{\mu}_{0} \times\left(\overleftarrow{\nabla^{\prime}}+i \mathbf{v} \omega\right)$

For the different orientations of the magnetic dipole, we carry out the algebra and find a result of the form of Eq. (4.4), or

$$
\begin{aligned}
\left(F_{x}\right)_{\mu}= & -\frac{\mu_{0}^{2}}{128 \pi^{2} a^{4}}\left(\gamma^{2}-1\right) \int_{0}^{\infty} d \hat{u} \hat{u}^{3} e^{-\hat{u}} \\
& \times \int_{0}^{2 \pi} d \theta \frac{\cos \theta}{1+\left(\gamma^{2}-1\right) \cos ^{2} \theta}\left(\tilde{f}^{E}+\tilde{f}^{H}\right) \\
& \times\left\{\begin{array}{cc}
\gamma^{2} \cos ^{2} \theta, & \boldsymbol{\mu}_{0}=\hat{\mathbf{x}} \mu_{0} \\
\sin ^{2} \theta, & \boldsymbol{\mu}_{0}=\hat{\mathbf{y}} \mu_{0} \\
1, & \boldsymbol{\mu}_{0}=\hat{\mathbf{z}} \mu_{0}
\end{array}\right.
\end{aligned}
$$

which looks just like Eq. (4.4) except that $\mathbf{d}_{0} \rightarrow \boldsymbol{\mu}_{0}$, and the $f$ 's are replaced by $\tilde{f}$ 's which are defined by

$$
\tilde{f}^{E}=\frac{\gamma^{2}}{\gamma^{2}-1} \frac{1}{\sin ^{2} \theta} f^{E}, \quad \tilde{f}^{H}=\sin ^{2} \theta \frac{\gamma^{2}-1}{\gamma^{2}} f^{H} .
$$

Now, because of the kinematic factors, the TE contribution dominates, and using the previously stated behaviors (4.7) for small $v$, we find

$$
\left(F_{x}^{x, y, z}\right)_{\mu}=-\frac{\mu_{0}^{2}}{128 \pi a^{4}} \frac{\hat{\alpha}^{2}}{8 \hat{\beta}} v(3,1,4), \quad v \ll \hat{\beta} \ll 1,
$$

since the angular integrals are the same as for the electric dipole. 
Again the electric dipole friction is relatively smaller than the corresponding electric charge case [2], by a factor of order $(d / e a)^{2}$.

\section{QUANTUM VACUUM FRICTION}

\section{A. $d d$ fluctuations}

Let us return to the situation of a particle moving uniformly in vacuum, but now quantize the dipole. We assume the particle has no mean dipole moment in its rest frame, $\left\langle\mathbf{d}^{\prime}\left(t^{\prime}\right)\right\rangle=0$. The formula we obtained in Sec. IIC for the force in the moving frame of the particle expressed in terms of the rest-frame dipole moments, Eq. (2.26), is quantized by using the fluctuation-dissipation theorem $[21,22]$

$$
\begin{gathered}
\left\langle\frac{\mathbf{d}^{\prime}\left(t_{1}^{\prime}\right) \mathbf{d}^{\prime}\left(t_{2}^{\prime}\right)+\mathbf{d}^{\prime}\left(t_{2}^{\prime}\right) \mathbf{d}^{\prime}\left(t_{1}^{\prime}\right)}{2}\right\rangle \equiv\left\langle\mathscr{S} \mathbf{d}^{\prime}\left(t_{1}^{\prime}\right) \mathbf{d}^{\prime}\left(t_{2}^{\prime}\right)\right\rangle \\
=\int_{-\infty}^{\infty} \frac{d v}{2 \pi} e^{-i v\left(t_{1}^{\prime}-t_{2}^{\prime}\right)} \operatorname{Im} \boldsymbol{\alpha}(v) \operatorname{coth} \frac{\beta^{\prime} v}{2},
\end{gathered}
$$

where we have symmetrized the dipole operators. Here, $\beta^{\prime}$ is the inverse temperature in the particle's rest frame and $\alpha$ is the electric polarizability tensor of the particle in its rest frame. We Fourier transform Eq. (5.1) and find the proper replacement to be made in Eq. (2.26) when quantizing the dipole is

$$
\left|\tilde{\mathbf{d}}^{\prime}(\omega)\right|^{2} \rightarrow T^{\prime} \operatorname{Im} \alpha(\omega) \operatorname{coth} \frac{\beta^{\prime} \omega}{2} .
$$

Here, $T^{\prime}$ is the total time that the configuration exists measured in the rest frame of the particle, related to $T$ by $T=$ $\gamma T^{\prime}$, because the change in the particle coordinate is $d x^{\prime}=0$. For an isotropic particle, $\boldsymbol{\alpha}=\alpha \mathbf{1}$, then, the force arising from the dipole fluctuations is

$$
\left(F_{x}\right)_{d d}=-\frac{v}{4 \pi^{2}} \int_{-\infty}^{\infty} d \omega \omega^{4} \operatorname{Im} \alpha(\omega) \operatorname{coth} \frac{\beta^{\prime} \omega}{2} .
$$

The above argument is somewhat heuristic. For a more rigorous approach, consider first the free energy in the rest frame of the particle, located at $\mathbf{r}_{\mathbf{1}}^{\prime}$ at time $t_{1}^{\prime}$, which may be written as ${ }^{4}$

$$
\begin{aligned}
\mathscr{F}^{\prime}\left(t_{1}^{\prime}\right)= & -\left\langle\mathbf{d}^{\prime}\left(t_{1}^{\prime}\right) \cdot \mathbf{E}^{\prime}\left(\mathbf{r}_{1}^{\prime}, t_{1}^{\prime}\right)\right\rangle \\
= & -\int_{-\infty}^{\infty} d t_{2}^{\prime} \int_{-\infty}^{\infty} \frac{d \omega}{2 \pi} e^{-i \omega\left(t_{1}^{\prime}-t_{2}^{\prime}\right)} \int \frac{\left(d \mathbf{k}_{\perp}^{\prime}\right)}{(2 \pi)^{2}} e^{i \mathbf{k}_{\perp}^{\prime} \cdot\left(\mathbf{r}_{1}^{\prime}-\mathbf{r}_{2}^{\prime}\right)_{\perp}} \\
& \times\left.\operatorname{trg}_{R}^{\prime}\left(z_{1}^{\prime}, z_{2}^{\prime} ; \mathbf{k}_{\perp}^{\prime}, \omega\right)\left\langle\mathscr{S} \mathbf{d}^{\prime}\left(t_{2}^{\prime}\right) \mathbf{d}^{\prime}\left(t_{1}^{\prime}\right)\right\rangle\right|_{\mathbf{r}_{1 \perp}^{\prime}=\mathbf{r}_{2 \perp}^{\prime}, z_{1}^{\prime}=z_{2}^{\prime}} .
\end{aligned}
$$

In the frame in which the particle is moving with velocity $\mathbf{v}=v \hat{\mathbf{x}}$, the transformed free energy is $\mathscr{F}=\frac{1}{\gamma} \mathscr{F}^{\prime} .^{5}$ The corresponding force on the particle may then be obtained by

\footnotetext{
${ }^{4}$ Here $R$ is an explicit acknowledgment that we are using the retarded Green's function as opposed to the thermal Green's function. For the relation between these Green's functions, see Appendix B.

${ }^{5}$ Note that the action $W=-\mathscr{F} T$ is Lorentz invariant, which is why $T$ and $\mathscr{F}$ transform contravariantly.
}

the insertion of a factor of $-i\left(k_{x}^{\prime}+v \omega\right)$ in Eq. (5.4). Using Eq. (5.1), we then have, in general,

$$
\begin{aligned}
\left(F_{x}\right)_{d d}= & -\int_{-\infty}^{\infty} \frac{d \omega}{2 \pi} \operatorname{tr} \operatorname{Im} \boldsymbol{\alpha}(\omega) \int \frac{\left(d \mathbf{k}_{\perp}^{\prime}\right)}{(2 \pi)^{2}}\left(k_{x}^{\prime}+v \omega\right) \\
& \times \operatorname{Im} \mathbf{g}_{R}^{\prime}\left(0,0 ; \mathbf{k}_{\perp}^{\prime}, \omega\right) \operatorname{coth} \frac{\beta^{\prime} \omega}{2} .
\end{aligned}
$$

The imaginary part of the Green's function emerges from the symmetry under $\omega \rightarrow-\omega$ and $k_{x}^{\prime} \rightarrow-k_{x}^{\prime}$. For an isotropic particle, the trace of $\mathbf{g}_{R}^{\prime}(0,0)$ is in vacuum $\omega^{2} / \kappa,{ }^{6}$ where $\kappa=\sqrt{k_{\perp}^{\prime 2}-\omega^{2}}$. This yields

$$
\begin{aligned}
\left(F_{x}\right)_{d d}= & -\frac{1}{8 \pi^{3}} \int_{-\infty}^{\infty} d \omega \omega^{2} \operatorname{Im} \alpha(\omega) \\
& \times \operatorname{coth} \frac{\beta^{\prime} \omega}{2} \int d k_{x}^{\prime} d k_{y}^{\prime} \operatorname{Im} \frac{1}{\kappa}\left(k_{x}^{\prime}+v \omega\right) .
\end{aligned}
$$

The imaginary part of $1 / \kappa$ arises from the branch line in $\kappa$, according to Eqs. (2.13) and (2.14). And then doing the $k_{x}$ integration over the interval from $-|\omega|$ to $|\omega|$ we are left with precisely Eq. (5.3).

\section{B. $E E$ fluctuations}

This is not the end of the story. We must now also include fluctuations in the electromagnetic field. This contribution arises from the force term

$$
\left(F_{x}\right)_{E E}=\frac{1}{\gamma} \int_{-\infty}^{\infty} \frac{d \omega}{2 \pi} \operatorname{tr} \boldsymbol{\alpha}(\omega)^{*} \nabla_{x}\left\langle\mathbf{E}^{\prime} \mathbf{E}^{\prime}\right\rangle(\omega),
$$

which represents the interaction between the dipoles induced by the field fluctuations. Here the factor of $1 / \gamma$ is present to transform the energy in the rest frame of the particle to that in the rest frame of the blackbody radiation. We will here assume once again that the field operators are to be merely symmetrized,

$$
\begin{aligned}
\left\langle\mathbf{E}^{\prime} \mathbf{E}^{\prime}\right\rangle\left(\mathbf{r}_{1}^{\prime}, \mathbf{r}_{2}^{\prime} ; \omega\right) \equiv & \int_{-\infty}^{\infty} d\left(t_{1}^{\prime}-t_{2}^{\prime}\right) e^{i \omega\left(t_{1}^{\prime}-t_{2}^{\prime}\right)} \\
& \times\left\langle\mathscr{S} \mathbf{E}^{\prime}\left(\mathbf{r}_{1}^{\prime}, t_{1}^{\prime}\right) \mathbf{E}^{\prime}\left(\mathbf{r}_{2}^{\prime}, t_{2}^{\prime}\right)\right\rangle .
\end{aligned}
$$

Here $\mathbf{E}^{\prime}\left(\mathbf{r}^{\prime}, t^{\prime}\right)$ is the electric field in the rest frame of the particle. We need to Lorentz transform the fields to the rest frame of the blackbody radiation in the vacuum, accomplished by using

$$
\begin{aligned}
& E_{x}^{\prime}\left(\mathbf{r}^{\prime}, t^{\prime}\right)=E_{x}(\mathbf{r}, t), \\
& E_{y}^{\prime}\left(\mathbf{r}^{\prime}, t^{\prime}\right)=\gamma\left[E_{y}(\mathbf{r}, t)-v B_{z}(\mathbf{r}, t)\right], \\
& E_{z}^{\prime}\left(\mathbf{r}^{\prime}, t^{\prime}\right)=\gamma\left[E_{z}(\mathbf{r}, t)+v B_{y}(\mathbf{r}, t)\right],
\end{aligned}
$$

where

$$
x^{\prime}=\gamma(x-v t), \quad y^{\prime}=y, \quad z^{\prime}=z, \quad t^{\prime}=\gamma(t-v x) .
$$

\footnotetext{
${ }^{6}$ The vacuum and the vacuum retarded Green's function are Lorentz invariant. On the other hand, if we thought of $\Gamma_{R}$ in terms of an expectation value of field products, those transform as described in the next subsection, with the same result.
} 
Using Maxwell's equations to eliminate $\mathbf{B}$ in favor of $\mathbf{E}$, that is, in the frequency domain $\nabla \times \mathbf{E}=-i \nu \mathbf{B}$ [corresponding to the sign of the Fourier transform in Eq. (5.11)], we can express the desired correlation function in the rest frame of the blackbody radiation using the fluctuation-dissipation theorem again:

$$
\left\langle\mathscr{S} \mathbf{E}\left(\mathbf{r}_{1}, t_{1}\right) \mathbf{E}\left(\mathbf{r}_{2}, t_{2}\right)\right\rangle=\int_{-\infty}^{\infty} \frac{d v}{2 \pi} e^{-i v\left(t_{1}-t_{2}\right)} \operatorname{Im} \boldsymbol{\Gamma}_{R}\left(\mathbf{r}_{1}, \mathbf{r}_{2} ; v\right) \operatorname{coth} \frac{\beta v}{2},
$$

where the retarded Green's dyadic $\boldsymbol{\Gamma}_{R}$ is given by Eqs. (2.6) and (2.7) and $\beta$ is the inverse temperature of the blackbody radiation.

Again, let us consider an isotropic particle, so the trace is taken over the field correlation function in the particle rest frame,

$$
\operatorname{tr}\left\langle\mathscr{S} \mathbf{E}^{\prime}\left(\mathbf{r}_{1}^{\prime}, t_{1}^{\prime}\right) \mathbf{E}^{\prime}\left(\mathbf{r}_{2}^{\prime}, t_{2}^{\prime}\right)\right\rangle=\int \frac{d v}{2 \pi} \frac{\left(d \mathbf{k}_{\perp}\right)}{(2 \pi)^{2}} e^{-i \gamma\left(\nu-k_{x} v\right)\left(t_{1}^{\prime}-t_{2}^{\prime}\right)} e^{i \gamma\left(k_{x}-v v\right)\left(x_{1}^{\prime}-x_{2}^{\prime}\right)} f\left(v, k_{x}\right) \operatorname{Im} \frac{1}{2 \kappa} \operatorname{coth} \frac{\beta v}{2},
$$

where a straightforward bit of algebra yields

$$
f\left(v, k_{x}\right)=2 \gamma^{2} v^{2}\left(\frac{v}{v}-k_{x}\right)^{2}
$$

Carrying out the frequency Fourier transform of Eq. (5.12) yields a $\delta$ function setting $v=\omega / \gamma+v k_{x}$, for which $f\left(\nu, k_{x}\right)=2 \omega^{2}$. The gradient in Eq. (5.7) supplies a factor of $i k_{x}$, since it refers to the blackbody rest frame. The imaginary part of the Green's dyadic arises again from carrying out the $k_{y}$ integral over the branch line of $1 / \kappa$, with the result

$$
\left(F_{x}\right)_{E E}=\frac{i}{8 \pi^{2} \gamma^{2}} \int_{-\infty}^{\infty} d \omega \omega^{2} \alpha(\omega)^{*} \int d k_{x} k_{x} \operatorname{sgn}\left(\frac{\omega}{\gamma}+v k_{x}\right) \operatorname{coth}\left[\frac{\beta}{2}\left(\frac{\omega}{\gamma}+v k_{x}\right)\right],
$$

where the limits on the $k_{x}$ integration are determined by the condition $k_{x}^{2}<v^{2}$. Then, with $k_{x}=u \omega$, we have

$$
\left(F_{x}\right)_{E E}=\frac{1}{8 \pi^{2} \gamma^{2}} \int_{-\infty}^{\infty} d \omega \omega^{4} \operatorname{Im} \alpha(\omega) \int_{-u_{-}}^{u_{+}} d u u \operatorname{coth}\left[\frac{\beta \omega}{2}\left(\frac{1}{\gamma}+v u\right)\right],
$$

where the limits on the $u$ integration are now given in terms of $u_{+}=\sqrt{\frac{1+v}{1-v}}$ and $u_{-}=\sqrt{\frac{1-v}{1+v}}$. Here, we have used the fact that $\alpha(\omega)^{*}=\alpha(-\omega)$. Thus only the imaginary part of $\alpha$ appears because otherwise the integrand is odd. Finally, on performing the $u$ integration, we obtain

$$
\begin{aligned}
\left(F_{x}\right)_{E E}= & \frac{1}{4 \pi^{2} v^{2} \beta^{2} \gamma^{2}} \int_{-\infty}^{\infty} d \omega \operatorname{Im} \alpha(\omega) \omega^{2}\left(\operatorname{Li}_{2}\left(e^{-\beta \omega_{-}}\right)-\operatorname{Li}_{2}\left(e^{-\beta \omega_{+}}\right)-v \beta^{2} \gamma^{2} \omega^{2}\right. \\
& \left.+v \beta\left\{\omega_{-} \ln \left[2 \sinh \left(\frac{\beta \omega_{-}}{2}\right)\right]+\omega_{+} \ln \left[2 \sinh \left(\frac{\beta \omega_{+}}{2}\right)\right]\right\}\right),
\end{aligned}
$$

where $\omega_{-}=\omega \sqrt{\frac{1-v}{1+v}}$ and $\omega_{+}=\omega \sqrt{\frac{1+v}{1-v}}$ are the corresponding Doppler-shifted frequencies and $\mathrm{Li}_{2}$ denotes the dilogarithm function. It is immediate that this expression is odd in $v$. That the integrand is even in $\omega$, which is already evident from Eq. (5.14), follows from the reflection property of the dilogarithm: $\operatorname{Li}_{2}\left(z^{-1}\right)=-\mathrm{Li}_{2}(z)-\frac{\pi^{2}}{6}-\frac{1}{2} \ln ^{2}(-z)$.

An alternative approach is to evaluate the ensemble average of the symmetrized field operators directly in the rest frame of the particle. This may be achieved by employing the fluctuation-dissipation theorem at the level of each $k_{x}^{\prime}$ Fourier component, using the corresponding Lorentz-transformed inverse temperature from Eq. (D4):

$$
\left\langle\mathscr{S} \mathbf{E}^{\prime}\left(\mathbf{r}_{1}^{\prime}, t_{1}^{\prime}\right) \mathbf{E}^{\prime}\left(\mathbf{r}_{2}^{\prime}, t_{2}^{\prime}\right)\right\rangle=\int_{-\infty}^{\infty} \frac{d \omega}{2 \pi} e^{-i \omega\left(t_{1}^{\prime}-t_{2}^{\prime}\right)} \int \frac{\left(d \mathbf{k}_{\perp}^{\prime}\right)}{(2 \pi)^{2}} e^{i \mathbf{k}_{\perp}^{\prime} \cdot\left(\mathbf{r}_{1}^{\prime}-\mathbf{r}_{2}^{\prime}\right)_{\perp}} \operatorname{Im} \boldsymbol{g}_{R}^{\prime}\left(z_{1}^{\prime}, z_{2}^{\prime} ; \mathbf{k}_{\perp}^{\prime}, \omega\right) \operatorname{coth}\left(\frac{\beta \gamma}{2}\left(\omega+v k_{x}^{\prime}\right)\right) .
$$

The analog of Eq. (5.5) is then generally

$$
\left(F_{x}\right)_{E E}=\int_{-\infty}^{\infty} \frac{d \omega}{2 \pi} \operatorname{tr} \operatorname{Im} \boldsymbol{\alpha}(\omega) \int \frac{\left(d \mathbf{k}_{\perp}^{\prime}\right)}{(2 \pi)^{2}}\left(k_{x}^{\prime}+v \omega\right) \operatorname{Im} \mathbf{g}_{R}^{\prime}\left(0,0 ; \mathbf{k}_{\perp}^{\prime}, \omega\right) \operatorname{coth}\left(\frac{\beta \gamma}{2}\left(\omega+v k_{x}^{\prime}\right)\right) .
$$

Here the imaginary part of the Green's function emerges as before, so for the isotropic case,

$$
\left(F_{x}\right)_{E E}=\frac{1}{8 \pi^{2}} \int_{-\infty}^{\infty} d \omega \operatorname{Im} \alpha(\omega) \omega^{2} \operatorname{sgn}(\omega) \int d k_{x}^{\prime}\left(k_{x}^{\prime}+v \omega\right) \operatorname{coth}\left(\frac{\beta \gamma}{2}\left(\omega+v k_{x}^{\prime}\right)\right),
$$

where the limits on the $k_{x}^{\prime}$ integration are determined by the condition $k_{x}^{\prime 2}<\omega^{2}$. This is equivalent to Eq. (5.14) as seen by making the substitution $k_{x}=\gamma\left(k_{x}^{\prime}+v \omega\right)$. 


\section{Einstein-Hopf effect}

The vacuum frictional forces (5.3) and (5.15) agree with those in Refs. [10,11,23,24], and references therein, except for a factor of $4 \pi$, due to the use of rationalized units in our case. The two terms are combined to yield the total vacuum frictional force

$$
F_{\mathrm{tot}}=F_{d d}+F_{E E}=-\frac{1}{4 \pi^{2} \gamma^{2}} \int_{0}^{\infty} d \omega \omega^{4} \operatorname{Im} \alpha(\omega) \int_{u_{-}}^{u_{+}} \frac{d y}{v} \frac{1}{v}\left(y-\frac{1}{\gamma}\right)\left[\operatorname{coth} \frac{\beta^{\prime} \omega}{2}-\operatorname{coth} \frac{\beta \omega y}{2}\right],
$$

which vanishes at zero temperature and at zero velocity. We might expect $\beta^{\prime}=\gamma \beta$, assuming $\beta$ transforms like the time component of a four-vector. ${ }^{7}$ This is further explored in Appendix D.

As pointed out in Ref. [11] this result is equivalent to the Einstein-Hopf effect, which refers to low velocities. That force may be written in terms of the blackbody spectral density $\rho(\omega)$ as (recall that the relation between the Heaviside-Lorentz and the more usual Gaussian units for polarizability is $\alpha_{\mathrm{HL}}=4 \pi \alpha_{\mathrm{G}}$ )

$$
F_{x}^{\mathrm{EH}}=-v \int_{0}^{\infty} d \omega \omega \operatorname{Im} \alpha(\omega)\left[\rho(\omega)-\frac{\omega}{3} \frac{d}{d \omega} \rho(\omega)\right] \text {. }
$$

For the Planck spectrum,

$$
\rho(\omega)=\frac{\omega^{3}}{2 \pi^{2}} \operatorname{coth} \frac{\beta \omega}{2} .
$$

Inserting this into Eq. (5.21) we have for the Einstein-Hopf friction [25]

$$
F_{x}^{\mathrm{EH}}=-\frac{2 v}{3 \pi} \frac{1}{4 \pi} \int_{0}^{\infty} d \omega \omega^{4} \operatorname{Im} \alpha(\omega) \frac{\beta \omega / 2}{\sinh ^{2} \beta \omega / 2} .
$$

This is exactly what is obtained from Eq. (5.20) if it is expanded for small $v$ when $\beta=\beta^{\prime}$.

It seems appropriate to conclude this section with a few remarks concerning numerical magnitudes. For example, suppose that the moving particle is a small gold nanosphere of radius $a$. The polarizability of such a particle is

$$
\alpha(\omega)=4 \pi a^{3} \frac{\varepsilon(\omega)-1}{\varepsilon(\omega)+2} .
$$

Let the permittivity be described by the Drude model (4.6). Then for low frequencies [also as seen in Ref. [11], since $v=$ $\omega_{p}^{2} /(4 \pi \sigma), \sigma$ being the conductivity]

$$
\operatorname{Im} \alpha(\omega) \approx 4 \pi a^{3} \frac{3 \omega \nu}{\omega_{p}^{2}} .
$$

Inserting this into Eq. (5.23), we obtain

$$
F^{\mathrm{EH}}=-v \frac{v}{a} \frac{1}{21 \pi} \frac{(2 \pi a / \beta)^{6}}{\left(\omega_{p} a\right)^{2}} \approx-v \times 10^{-23} \mathrm{~N},
$$

where we used values appropriate for gold, $\omega_{p}=9 \mathrm{eV}$ and $v=0.035 \mathrm{eV}$, and considered room temperature $\beta=40 \mathrm{eV}^{-1}$ and a sphere of radius $a=100 \mathrm{~nm}$. This is apparently beyond experimental reach, as we see by comparing with the usual

\footnotetext{
${ }^{7}$ However, Ref. [11] suggests a different, model-dependent, velocity dependence in equilibrium as we will explore elsewhere. In any case, for low velocities, $\beta^{\prime}$ should be $\beta$.
}

Casimir-Polder force for a perfectly conducting sphere of radius $a$ a distance $d$ above a conducting plate,

$$
F^{\mathrm{CP}}=-\frac{3 \alpha_{\mathrm{G}}}{2 \pi d^{5}}=-\frac{3 a^{3}}{2 \pi d^{5}} \sim 10^{-17} \mathrm{~N},
$$

for $a=100 \mathrm{~nm}$ and $d=1000 \mathrm{~nm}$, which is already quite small. [If we had used a radiation reaction model instead, so $\operatorname{Im} \alpha(\omega)=\frac{1}{6 \pi} \omega^{3} \alpha_{0}^{2}$, as in Ref. [11], a much smaller value than that in Eq. (5.26) would result.]

Finally, we note that the integrand in Eq. (5.20) is not positive definite. Nevertheless, we would expect the frictional force to always be negative, at least when $\operatorname{Im} \alpha(\omega)$ is a power function of $\omega$. We illustrate this numerically in Fig. 1, which plots the dimensionless integral

$$
I_{n}\left(\beta / \beta^{\prime}, v\right)=\int_{0}^{\infty} d x x^{4+n} f\left(x, \beta / \beta^{\prime}, v\right),
$$

where

$$
\begin{aligned}
f\left(x, \beta / \beta^{\prime}, v\right)= & \frac{1}{v^{2}} \int_{u_{-}}^{u_{+}} d y\left(y-\sqrt{1-v^{2}}\right) \\
& \times\left[\operatorname{coth} \frac{x}{2}-\operatorname{coth} \frac{\beta x y}{2 \beta^{\prime}}\right] .
\end{aligned}
$$

Here for the "nanosphere" model (5.25) $n=1$, while for the radiation-reaction model $n=3$. To convert these integrals into frictional forces, we have the following expressions for

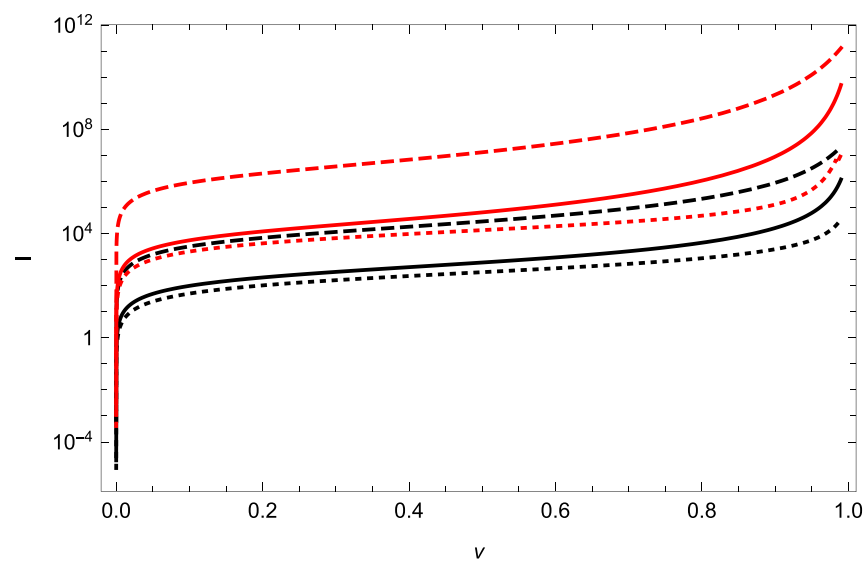

FIG. 1. Quantum frictional force written in the form of the dimensionless integral (5.28) plotted as a function of the velocity $v$. The lower set of curves (black) is for $n=1$ and the upper set (red) is for $n=3$. In each case, three situations are envisaged: $\beta=\beta^{\prime}$ (solid), $\beta=\beta^{\prime} / 2$ (dashed), and $\beta=2 \beta^{\prime}$ (dotted). 
the two models:

$$
\left(F_{x}\right)^{R R}=-\frac{1}{6 \pi^{2} \gamma^{2}} \frac{\alpha_{0}^{2}}{\beta^{\prime 8}} I_{3}, \quad\left(F_{x}\right)^{N S}=-\frac{3}{\pi} \frac{v}{a \gamma^{2}} \frac{\left(a / \beta^{\prime}\right)^{6}}{\left(\omega_{p} a\right)^{2}} I_{1} .
$$

In Appendix E we show that $I_{n}$ is expressible in closed form, and always positive. Further exploration of the sign of $F$ will appear elsewhere.

\section{CONCLUSIONS}

What is remarkable about the straightforward calculations sketched in this paper is that starting simply from Maxwell's equations, and the corresponding Lorentz force law, familiar in elementary physics, one deduces the formula for the energy emitted by dipole radiation, without ever invoking radiation fields or the concept of radiation reaction. This analysis may appear similar to the discussion of friction due to thermal [3] or zero-point [4] fluctuations, in which a dipole, including radiation reaction, is coupled to fluctuating electromagnetic fields. In the latter case, of course, no force on the dipole is found at zero temperature [26,27]. The classical theory does not involve fluctuations. Our results generalize those of Ref. [5].

To demonstrate that our considerations are sensible, we recover known results for the spectral distribution of the Vavilov-Čerenkov radiation emitted by a superluminal electric or magnetic dipole. In the latter case, we confirm, in accordance with Refs. [7,16], that there is a discontinuity in the radiation at threshold, $v n(\omega)=1$, for polarization of the magnetic dipole perpendicular to the direction of motion.

We then present a discussion of the classical electromagnetic friction experienced by a time-independent electric or magnetic dipole moving parallel to an imperfectly conducting surface. We derive general formulas valid for all velocities and polarizations. Explicit results are given for the low velocity region, $v \ll a v$, where $v$ is the damping parameter, proportional to the resistivity of the conductor, and $a$ is the distance of the dipole from the surface of the conductor. This is likely the regime in which this friction might be more accessible to experiment. Again, our results are more general and more simply derived that those given previously [20].

We finally show how by minimal use of the fluctuationdissipation theorem applied to both dipoles and fields we can recover the Einstein-Hopf effect, which, for arbitrary veloc- ities, yields the quantum blackbody friction in vacuum for a polarizable particle found by Dedkov and Kyasov [10] and Volokitin and Persson [11]. In Appendix $C$ we show that the same considerations give rise to the usual Casimir-Polder interaction with an arbitrary body. In subsequent publications, we will further investigate quantum friction in the vicinity of other bodies, and explore the energetics that can lead to nonequilibrium stationary configurations [28].

\section{ACKNOWLEDGMENTS}

We thank our collaborators P. Parashar and S. Fulling for many insightful comments on this work. This research was supported in part by the US National Science Foundation, Grant No. 1707511.

\section{APPENDIX A: LORENTZ TRANSFORMATION OF CHARGE AND CURRENT DENSITIES}

Since it appears a bit subtle for a time-dependent dipole, we sketch the inference of the charge and current density for a moving dipole given in Eq. (2.2) from that for a stationary dipole,

$$
\rho^{\prime}\left(\mathbf{r}^{\prime}, t^{\prime}\right)=-\mathbf{d}^{\prime}\left(t^{\prime}\right) \cdot \nabla^{\prime} \delta\left(\mathbf{r}^{\prime}\right), \quad \mathbf{j}^{\prime}\left(\mathbf{r}^{\prime}, t^{\prime}\right)=\dot{\mathbf{d}}^{\prime}\left(t^{\prime}\right) \delta\left(\mathbf{r}^{\prime}\right) .
$$

Recall that unprimed quantities refer to the frame where the particle has velocity $\mathbf{v}=\hat{\mathbf{x}} v$, while primed quantities refer to the particle's rest frame. The dot always means derivative with respect to the argument. The coordinates in the two frames are related by

$$
x=\gamma\left(x^{\prime}+v t^{\prime}\right), \quad t=\gamma\left(t^{\prime}+v x^{\prime}\right), \quad y=y^{\prime}, \quad z=z^{\prime} .
$$

Now $(\rho, \mathbf{j})$ constitutes a four-vector, so

$$
\begin{aligned}
\rho(\mathbf{r}, t)= & \gamma\left[\rho^{\prime}\left(\mathbf{r}^{\prime}, t^{\prime}\right)+v j_{x}^{\prime}\left(\mathbf{r}^{\prime}, t^{\prime}\right)\right] \\
= & \gamma\left[-d_{x}^{\prime}(\gamma(t-v x)) \gamma\left(\partial_{x}+v \partial_{t}\right)-\mathbf{d}_{\perp}^{\prime}(\gamma(t-v x)) \cdot \nabla_{\perp}\right. \\
& \left.+v \dot{d}_{x}^{\prime}(\gamma(t-v x))\right] \delta(\gamma(x-v t)) \delta\left(\mathbf{x}_{\perp}\right),
\end{aligned}
$$

where the $\perp$ sign signifies the $y, z$ directions. We use the identity

$$
f(x) \partial_{x} \delta(x)=f(0) \partial_{x} \delta(x)-\delta(x) \partial_{x} f(0)
$$

to write the above as

$$
\begin{aligned}
\rho(\mathbf{r}, t)=[- & \frac{1}{\gamma} d_{x}^{\prime}\left(\frac{t}{\gamma}\right) \partial_{x} \delta(x-v t)+\frac{1}{\gamma} \delta(x-v t) \partial_{x} d_{x}^{\prime}(\gamma(t-v x))-\mathbf{d}_{\perp}^{\prime}\left(\frac{t}{\gamma}\right) \cdot \nabla_{\perp} \delta(x-v t) \\
& \left.+v \dot{d}_{x}^{\prime}(\gamma(t-v x)) \delta(x-v t)\right] \delta(y) \delta(z)=-\mathbf{d}(t) \cdot \nabla \delta(x-v t) \delta(y) \delta(z),
\end{aligned}
$$

which uses the transformation properties for the dipole moments, Eq. (2.24), and notes that the second and fourth terms in the square brackets cancel.

The $x$ component of the current,

$$
\begin{aligned}
j_{x}(\mathbf{r}, t) & =\gamma\left[j_{x}^{\prime}\left(\mathbf{r}^{\prime}, t^{\prime}\right)+v \rho^{\prime}\left(\mathbf{r}^{\prime}, t^{\prime}\right)\right] \\
& =\left[\dot{d}_{x}^{\prime}(\gamma(t-v x))-v d_{x}^{\prime}(\gamma(t-v x)) \gamma\left(\partial_{x}+v \partial_{t}\right)-v \mathbf{d}_{\perp}^{\prime}(\gamma(t-v x)) \cdot \nabla_{\perp}\right] \delta(x-v t) \delta(y) \delta(z),
\end{aligned}
$$


becomes, using the identity (A4),

$$
j_{x}(\mathbf{r}, t)=\left\{\dot{d}_{x}^{\prime}(\gamma(t-v x))-\frac{v}{\gamma}\left[d_{x}^{\prime}\left(\frac{t}{\gamma}\right) \partial_{x}+v \partial_{t} d_{x}^{\prime}(\gamma(t-v x))\right]-v \mathbf{d}_{\perp}^{\prime}\left(\frac{t}{\gamma}\right) \cdot \nabla_{\perp}\right\} \delta(x-v t) \delta(y) \delta(z) .
$$

The first and third terms here combine to give $\frac{1}{\gamma^{2}} \dot{d}_{x}^{\prime}(\gamma(t-$ $v x)$ ) so when we use the transformation properties (2.24) we obtain the expected result,

$$
j_{x}(\mathbf{r}, t)=\left[-v \mathbf{d}(t) \cdot \nabla+\dot{d}_{x}(t)\right] \delta(x-v t) \delta(y) \delta(z) .
$$

More immediately, the $y$ component of the current is transformed to

$$
j_{y}(\mathbf{r}, t)=\dot{d}_{y}(t) \delta(x-v t) \delta(y) \delta(z) .
$$

Thus the charge and current densities due to an electric dipole in the moving frame of the particle, Eq. (2.2), are derived from the rest-frame form. A similar argument applies for the charge and current densities due to a magnetic dipole, Eq. (2.30).

\section{APPENDIX B: RELATION BETWEEN GREEN'S FUNCTIONS}

In Sec. V we used the symmetrized correlation function of the fields given in terms of the imaginary part of the retarded Green function by the fluctuation-dissipation theorem, with $n_{v}=\left(e^{\beta v}-1\right)^{-1}$ :

$$
\begin{aligned}
& \left\langle\mathscr{S} \mathbf{E}\left(\mathbf{r}_{1}, t_{1}\right) \mathbf{E}\left(\mathbf{r}_{2}, t_{2}\right)\right\rangle \\
& \quad=\int_{-\infty}^{\infty} \frac{d v}{2 \pi} \operatorname{Im} \boldsymbol{\Gamma}_{R}\left(\mathbf{r}, \mathbf{r}^{\prime} ; v\right)\left(2 n_{v}+1\right) e^{-i v\left(t_{1}-t_{2}\right)} .
\end{aligned}
$$

In quantum field theory we usually use the time-ordered product,

$$
\begin{aligned}
\left\langle\mathscr{T} \mathbf{E}\left(\mathbf{r}_{1}, t_{1}\right) \mathbf{E}\left(\mathbf{r}_{2}, t_{2}\right)\right\rangle= & \int_{-\infty}^{\infty} \frac{d v}{\pi} \operatorname{Im} \boldsymbol{\Gamma}_{R}\left(\mathbf{r}_{1}, \mathbf{r}_{2} ; v\right)\left(n_{v}+1\right) \\
& \times e^{-i v\left|t_{1}-t_{2}\right|} .
\end{aligned}
$$

The Fourier transform of this gives the thermal Green's function

$$
\begin{aligned}
\boldsymbol{\Gamma}_{+\beta}\left(\mathbf{r}_{1}, \mathbf{r}_{2} ; \omega\right)= & i\langle\mathscr{T} \mathbf{E E}\rangle\left(\mathbf{r}_{1}, \mathbf{r}_{2} ; \omega\right) \\
& =\frac{2}{\pi} \int_{-\infty}^{\infty} d v v\left(n_{v}+1\right) \frac{\operatorname{Im} \boldsymbol{\Gamma}_{R}\left(\mathbf{r}_{1}, \mathbf{r}_{2} ; v\right)}{(v-i \epsilon)^{2}-\omega^{2}}
\end{aligned}
$$

This is to be contrasted with the representation for the retarded Green's function, which has the form of a Kramers-Kronig relation,

$$
\boldsymbol{\Gamma}_{R}\left(\mathbf{r}_{1}, \mathbf{r}_{2} ; \omega\right)=\frac{2}{\pi} \int_{0}^{\infty} d v v \frac{\operatorname{Im} \boldsymbol{\Gamma}_{R}\left(\mathbf{r}_{1}, \mathbf{r}_{2} ; \nu\right)}{v^{2}-(\omega+i \epsilon)^{2}},
$$

which has no temperature dependence. The relation between the imaginary parts is

$$
\begin{aligned}
\operatorname{Im} \boldsymbol{\Gamma}_{+\beta}\left(\mathbf{r}_{1}, \mathbf{r}_{2} ; \omega\right) & =\operatorname{Im} \boldsymbol{\Gamma}_{R}\left(\mathbf{r}_{1}, \mathbf{r}_{2} ; \omega\right)\left(2 n_{\omega}+1\right), \\
2 n_{\omega}+1 & =\operatorname{coth} \frac{\beta \omega}{2},
\end{aligned}
$$

while the real parts are the same,

$$
\operatorname{Re} \boldsymbol{\Gamma}_{+\beta}\left(\mathbf{r}_{1}, \mathbf{r}_{2} ; \omega\right)=\operatorname{Re} \boldsymbol{\Gamma}_{R}\left(\mathbf{r}_{1}, \mathbf{r}_{2} ; \omega\right) .
$$

It is most usual to evaluate Casimir energies by integrating over Euclidean frequencies, $\omega \rightarrow i \zeta$. In that case, the Green's functions are identical:

$$
\begin{aligned}
\boldsymbol{\Gamma}_{+\beta}(i \zeta) & =\frac{2}{\pi} \int_{-\infty}^{\infty} d v v \frac{\operatorname{Im} \boldsymbol{\Gamma}_{R}(v)}{v^{2}+\zeta^{2}}\left(n_{v}+1\right) \\
& =\frac{2}{\pi} \int_{0}^{\infty} d v v \frac{\operatorname{Im} \boldsymbol{\Gamma}_{R}(v)}{v^{2}+\zeta^{2}}=\Gamma_{R}(i \zeta) .
\end{aligned}
$$

\section{APPENDIX C: STATIC CASIMIR-POLDER ENERGY}

Consider the static Casimir-Polder interaction between some (unspecified) background object and a polarizable atom. The contribution to the interaction free energy due to field fluctuations is

$$
\begin{aligned}
\mathscr{F}_{E E} & =-\frac{1}{2} \operatorname{tr} \int_{-\infty}^{\infty} \frac{d \omega}{2 \pi} \boldsymbol{\alpha}(\omega)^{*}\langle\mathscr{S} \mathbf{E E}\rangle(\omega) \\
& =-\frac{1}{2} \int_{-\infty}^{\infty} \frac{d \omega}{2 \pi} \operatorname{tr} \operatorname{Re} \boldsymbol{\alpha}(\omega) \operatorname{Im} \boldsymbol{\Gamma}_{R}(\omega) \operatorname{coth} \frac{\beta \omega}{2},
\end{aligned}
$$

since the imaginary part of the polarizability is odd. The second contribution to the energy comes from the dipole fluctuations, that is

$$
\mathscr{F}_{d d}=-\frac{1}{2 T} \operatorname{Tr} \mathbf{j}^{*} \frac{1}{\omega^{2}} \mathbf{\Gamma} \mathbf{j}
$$

which follows from Eq. (2.9). This immediately leads to, upon use of the current for a stationary dipole $\mathbf{j}(\mathbf{r}, \omega)=$ $-i \omega \mathbf{d}(\omega) \delta(\mathbf{r})$,

$$
\begin{aligned}
\mathscr{F}_{d d} & =-\frac{1}{2 T} \int_{-\infty}^{\infty} \frac{d \omega}{2 \pi}\left\langle\mathscr{S} \mathbf{d}(\omega)^{*} \cdot \boldsymbol{\Gamma}_{R}(\omega) \cdot \mathbf{d}(\omega)\right\rangle \\
& =-\frac{1}{2} \int_{-\infty}^{\infty} \frac{d \omega}{2 \pi} \operatorname{coth} \frac{\beta \omega}{2} \operatorname{tr} \operatorname{Im} \boldsymbol{\alpha}(\omega) \cdot \operatorname{Re} \boldsymbol{\Gamma}_{R}(\omega) .
\end{aligned}
$$

Thus the total free energy is just as expected:

$$
\begin{aligned}
\mathscr{F}_{C P} & =\mathscr{F}_{d d}+\mathscr{F}_{E E} \\
& =-\frac{1}{2} \int_{-\infty}^{\infty} \frac{d \omega}{2 \pi} \operatorname{coth} \frac{\beta \omega}{2} \operatorname{Im} \operatorname{tr}\left[\boldsymbol{\alpha}(\omega) \boldsymbol{\Gamma}_{R}(\omega)\right] .
\end{aligned}
$$

This result could be recaptured using the time-ordered polarizability and the thermal Green's function by use of Eq. (B5a) and Eq. (B5b),

$$
\mathscr{F}_{C P}=-\frac{1}{2} \int_{-\infty}^{\infty} \frac{d \omega}{2 \pi} \operatorname{Im} \operatorname{tr}\left[\boldsymbol{\alpha}_{+\beta}(\omega) \boldsymbol{\Gamma}_{+\beta}(\omega)\right],
$$

where

$$
\boldsymbol{\alpha}_{+\beta}(\omega)=i\langle\mathscr{T} \mathbf{d d}\rangle(\omega)=\frac{2}{\pi} \int_{-\infty}^{\infty} d \nu \frac{v\left(n_{\nu}+1\right) \operatorname{Im} \boldsymbol{\alpha}(\nu)}{(\nu-i \epsilon)^{2}-\omega^{2}} .
$$


The form in Eq. (C4) is perhaps more familiarly expressed in terms of Euclidean frequencies. Since the retarded Green's function and the polarizability have no singularities in the upper half $\omega$ plane, the contour of integration can be distorted to one encircling the positive imaginary axis, and then, accounting for the poles of the cotangent along that axis, we obtain

$$
\mathscr{F}_{C P}=-\frac{1}{\beta} \sum_{n=0}^{\infty} \operatorname{tr} \boldsymbol{\alpha}\left(i \zeta_{n}\right) \boldsymbol{\Gamma}\left(i \zeta_{n}\right),
$$

in terms of the Matsubara frequency $\zeta_{n}=2 \pi n / \beta$. (The prime on the summation sign means that the $n=0$ term is counted with half weight.)

\section{APPENDIX D: TRANSFORMATION OF BLACKBODY SPECTRAL DENSITY}

It was shown by Ford and O'Connell [29] that the spectral density (5.22) becomes in a frame moving with velocity $\mathbf{v}$

$$
\rho^{\prime}\left(\omega^{\prime}, \mathbf{k}^{\prime}\right)=\frac{\omega^{\prime 3}}{2 \pi^{2}} \operatorname{coth}\left[\frac{\beta}{2} \gamma \omega^{\prime}\left(1+\hat{\mathbf{k}}^{\prime} \cdot \mathbf{v}\right)\right] .
$$

Here $\beta$ is the inverse temperature in the blackbody rest frame. This is, in fact, just what we would expect if $\beta$ is thought to be the time component of four-vector, $\beta=\beta_{0}, \boldsymbol{\beta}=0$. So we could write

$$
\rho(\omega)=\rho(\omega, \mathbf{k})=\frac{\omega^{3}}{2 \pi^{2}} \operatorname{coth} \frac{1}{2} \beta_{\mu} k^{\mu}, \quad k^{\mu}=(\omega, \mathbf{k}) .
$$

Indeed this would become

$$
\rho^{\prime}\left(\omega^{\prime}, \mathbf{k}^{\prime}\right)=\frac{\omega^{\prime 3}}{2 \pi^{2}} \operatorname{coth} \frac{1}{2} \beta_{\mu}^{\prime} k^{\prime \mu}
$$

where, for a boost in the $x$ direction,

$$
\beta_{\mu}^{\prime} k^{\prime \mu}=\gamma \beta \omega^{\prime}+\gamma v \beta k_{x}^{\prime}=\gamma \beta \omega^{\prime}\left(1+v \frac{k_{x}^{\prime}}{k^{\prime}}\right), \quad k^{\prime}=\omega^{\prime} .
$$

So the result of Ford and O'Connell corresponds to the expected transformation of $\beta_{\mu}$.

\section{APPENDIX E: EVALUATION OF THE INTEGRAL $\boldsymbol{I}_{\boldsymbol{n}}$}

To evaluate the integral $I_{n}$ we carry out the $x$ integration first, with the result

$$
\begin{aligned}
I_{n}= & \Gamma(5+n) \zeta(5+n) \frac{1}{v^{2}} \int_{u_{-}}^{u_{+}} d y\left(y-\sqrt{1-v^{2}}\right) \\
& \times\left[1-\left(\frac{\beta}{\beta^{\prime}} y\right)^{-5-n}\right],
\end{aligned}
$$

and then we obtain

$$
\begin{aligned}
I_{n}= & 2 \Gamma(5+n) \zeta(5+n)\left\{2 v \gamma^{2}+\frac{1}{(3+n)(4+n)} \frac{\gamma^{3+n}}{v^{2}}\left(\frac{\beta^{\prime}}{\beta}\right)^{5+n}\right. \\
& \left.\times\left\{(1-v)^{3+n}-(1+v)^{3+n}+(3+n) v\left[(1-v)^{3+n}+(1+v)^{3+n}\right]\right\}\right\} .
\end{aligned}
$$

Since the last line above consists only of odd powers in $v$, all with positive coefficients for integer $n>-3$, both it and $I_{n}$ are positive.

[1] K. A. Milton, J. S. Høye, and I. Brevik, The reality of Casimir friction, Symmetry 8, 29 (2016).

[2] K. A. Milton, Y. Li, X. Guo, and G. Kennedy, Electrodynamic friction of a charged particle passing a conducting plate, Phys. Rev. Research 2, 023114 (2020).

[3] A. Einstein and L. Hopf, Statistische Untersuchung der Bewegung eines Resonators in einem Strahlungsfeld, Ann. Phys. (Leipzig) 338, 1105 (1910).

[4] T. H. Boyer, Derivation of the blackbody radiation spectrum without quantum assumptions, Phys. Rev. 182, 1374 (1969).

[5] M. Sonnleitner, N. Trautmann, and S. M. Barnett, Will a Decaying Atom Feel a Friction Force? Phys. Rev. Lett. 118, 053601 (2017).

[6] I. M. Frank, Doppler effect in a refractive medium, Izv. Akad. Nauk SSSR 6, 3 (1942).

[7] I. M. Frank, Vavilov-Cherenkov radiation for electric and magnetic multipoles, Usp. Fiz. Nauk 144, 251 (1984) [Sov. Phys. Usp. 27, 772 (1984)].
[8] V. L. Ginzburg, Radiation by uniformly moving sources, in Progress in Optics XXXII, edited by E. Wolf (Elsevier, Amsterdam, 1993), Chap. V.

[9] M. G. Silveirinha, Theory of quantum friction, New J. Phys. 16, 063011 (2014).

[10] G. V. Dedkov and A. A. Kyasov, Radiation of a neutral polarizable particle moving uniformly through a thermal radiation field, Phys. Scr. 89, 105501 (2014).

[11] A. I. Volokitin and B. N. J. Persson, Electromagnetic Fluctuations at the Nanoscale (Springer, Berlin, 2017), Chap. 8.

[12] J. Schwinger, Particles, Sources, and Fields (Addison-Wesley, Reading, MA, 1970).

[13] J. Schwinger, L. L. DeRaad, Jr., K. A. Milton, and W.-y. Tsai, Classical Electrodynamics (Perseus/Westview, Taylor and Francis, London, 1998).

[14] P. A. Cherenkov, Visible emission of clean liquids by action of $\gamma$ radiation, Dokl. Akad. Nauk SSSR 2, 451 (1934).

[15] I. E. Tamm and I. M. Frank, Coherent radiation of fast electrons in a medium, Dokl. Akad. Nauk SSSR 14, 107 (1937). 
[16] U. Leonhardt and Y. Rosenberg, Cherenkov radiation of light bullets, Phys. Rev. A 100, 063802 (2019).

[17] K. A. Milton and J. Schwinger, Electromagnetic Radiation: Variational Methods, Waveguides and Accelerators (Springer, Berlin, 2006).

[18] V. L. Ginzburg, Certain theoretical aspects of radiation due to superluminal motion in a medium, Sov. Phys. Usp. 2, 874 (1960).

[19] V. L. Ginzburg, Fields and radiation of 'true' and current dipoles, Radiophys. Quantum Electron. 27, 601 (1984).

[20] M. S. Tomassone and A. Widom, Electronic friction forces on molecules moving near metals, Phys. Rev. B 56, 4938 (1997).

[21] G. W. Ford, The fluctuation-dissipation theorem, Contemp. Phys. 58, 244 (2017).

[22] R. Kubo, The fluctuation-dissipation theorem, Rep. Prog. Phys. 29, 255 (1966).

[23] A. I. Volokitin, Friction force at the motion of a small relativistic neutral particle with respect to blackbody radiation,
Pis'ma Zh. Eksp. Fiz. 101, 479 (2015) [JETP Lett. 101, 427 (2015)].

[24] F. Intravaia, C. Henkel, and M. Antezza, Fluctuation-induced forces between atoms and surfaces: The Casimir-Polder interaction, in Casimir Physics, Lecture Notes in Physics Vol. 834, edited by D. A. R. Dalvit, P. W. Milonni, D. Roberts, and F. Da Rosa (Springer, Berlin, 2011), pp. 345-391.

[25] V. Mkrtchian, V. A. Parsegian, R. Podgornik, and W. M. Saslow, Universal Thermal Radiation Drag on Neutral Objects, Phys. Rev. Lett. 91, 220801 (2003).

[26] P. W. Milonni, Quantum mechanics of the Einstein-Hopf model, Am. J. Phys. 49, 177 (1981).

[27] P. W. Milonni, The Quantum Vacuum: An Introduction to Quantum Electrodynamics (Academic Press, Boston, 1994).

[28] D. Reiche, F. Intravaia, J.-T. Hsiang, K. Busch, and B. L. Hu, Nonequilibrium thermodynamics of quantum friction, Phys. Rev. A 102, 050203(R) (2020).

[29] G. W. Ford and R. F. O'Connell, Lorentz transformation of blackbody radiation, Phys. Rev. E 88, 044101 (2013). 